\section{Effect of Mutual Coupling on the Interference Rejection Capabilities of Linear and Circular Arrays in CDMA Systems}

\author{
Salman Durrani and Marek E. Bialkowski
}

\begin{abstract}
This paper is concerned with assessing the interference rejection capabilities of linear and circular array of dipoles that can be part of a base station of a code-division multiple-access cellular communication system. The performance criteria for signal-to-interference ratio (SIR) improvement employed in this paper is the spatial interference suppression coefficient. We first derive an expression for this figure of merit and then analyze and compare the SIR performance of the two types of arrays. For a linear array, we quantitatively assess the degradation in SIR performance as we move from array broadside to array end-fire direction. In addition, the effect of mutual coupling is taken into account.
\end{abstract}

Index Terms-Adaptive arrays, code-division multiple access, interference suppression, mutual coupling.

\section{INTRODUCTION}

Spatial filtering methods using advanced antenna techniques, smart or adaptive antennas, have received much attention over the last few years [1], [2]. Filtering in the spatial domain can separate spectrally and temporally overlapping signals from multiple mobile users, and hence the performance of a system can be significantly improved. Particular interest in such adaptive antennas has been shown with regard to codedivision multiple-access (CDMA) systems. This is because the thirdgeneration cellular networks, e.g., cdma2000 in North America and wide-band CDMA in Europe and Japan, are based on CDMA.

In CDMA systems, all users communicate simultaneously in the same frequency band, and hence multiple-access interference (MAI) is one of the major causes of transmission impairment. The interference rejection or signal-to-interference ratio (SIR) improvement capability is, therefore, an important measure of performance for an array antenna at a base station of a CDMA-based cellular system. This discrimination ability is in general a function of the number of antenna elements (including their spacing and geometry) and the direction of signal arrival of the desired user and the interferers. It is defined as a reciprocal of the spatial interference suppression coefficient, which is determined as an average cross-correlation between the beamforming weight vector toward for a desired user and the array steering vector toward the interferer [3]. The subject of interference suppression in general from a radar point of view was analyzed in [4]. In the context of CDMA systems, many research papers have addressed the SIR improvement in passing only, while also neglecting mutual coupling between antenna elements [3], [5]-[7]. The applications of the spatial interference suppression coefficient have cropped up in a number of recent papers and are mainly concerned with finding the mean value, e.g., in [8], where it is employed in determining an expression for the theoretical bit error rate of a smart antenna system, and in [9] and [10], where it is used to find the capacity of a multiantenna system for CDMA.

Manuscript received October 2, 2002; revised May 14, 2003. The work of S. Durrani was supported by an International Postgraduate Research Scholarship.

S. Durrani is with the School of Information Technology and Electrical Engineering, The University of Queensland, Brisbane Qld 4072 Australia (e-mail: dsalman@ieee.org).

M. E. Bialkowski is with the School of Information Technology and Electrical Engineering, The University of Queensland, Brisbane Qld 4072 Australia, and the Department of Electrical and Computer Engineering, National University of Singapore, 119260 Singapore (e-mail: bialkowski@ieee.org).

Digital Object Identifier 10.1109/TAP.2004.825640
In real arrays, mutual coupling is always present, and hence it is important to assess the SIR performance when mutual coupling is included in the array analysis. A common assumption in the study of mutual coupling is that it will lead to degradation in the performance of the system. However, this is not the case in general, e.g., it was found in [11] that by decreasing the amount of correlation between parallel channel, mutual coupling can in fact increase the channel capacity for multiple-input multiple-output (MIMO) systems.

Cellular base stations are also not restricted to linear array configurations. Before devising any beamforming algorithm, it is worthwhile to consider whether a chosen array configuration will enable optimal performance. Hence it is important to provide an assessment of performance for other configurations of arrays.

The main contributions of this paper are:

1) the derivation of expression of the spatial interference suppression coefficient for CDMA systems;

2) analysis and comparison of the SIR performance of linear and circular array geometries;

3) inclusion of the effect of mutual coupling on the SIR performance.

\section{Signal Model}

Consider a linear array of $N$ antenna elements located at the origin of the cartesian coordinate system and spaced a uniform distance $d=\lambda / 2$ along the $x$-axis. The array receives signals from $K$ narrow-band mobile users, which are randomly distributed in the $x y$-plane (azimuthal direction) in the far field of the array. In this case, the parameter that characterizes the location of the source is its angle of arrival (AOA) $\theta$, which is conventionally measured from the array broadside. We refer to $\theta=0^{\circ}$ as the broadside direction and $\theta=90^{\circ}$ as the end-fire direction. For simplicity, we first ignore mutual coupling and consider the linear array to be made of omnidirectional antenna elements. Selecting the first element as the phase reference, the received signal at the $n$th antenna element can be expressed as

$$
x_{n}(t)=\sum_{k=1}^{K} s_{k}(t) e^{-j \mathcal{K} d(n-1) \sin \theta_{k}}+n_{n}(t)
$$

where $\mathcal{K}$ is the wave number $=2 \pi / \lambda, \lambda=$ wavelength of the carrier frequency of the signals, $d=$ uniform interelement distance, $s_{k}(t)=$ signal transmitted by the $k$ th source as received by the reference antenna, $\theta_{k}=$ arrival angle of the $k$ th source as measured from the array broadside, and $n_{n}(t)=$ additive white Gaussian noise at the antenna elements with zero mean and having variance $\sigma^{2}$. Using vector notation, the received signal can be expressed as

$$
\mathbf{x}(t)=\sum_{k=1}^{K} \mathbf{a}\left(\theta_{k}\right) s_{k}(t)+\mathbf{n}(t)=\mathbf{A}(\theta) \mathbf{s}(t)+\mathbf{n}(t)
$$

where $\mathbf{x}(t)$ is an $N \times 1$ vector of measured voltages, $\mathbf{s}(t)$ is a $K \times 1$ signal vector, $\mathbf{n}(t)$ is an $N \times 1$ noise vector, and $\mathbf{A}(\theta)=\left[\mathbf{a}\left(\theta_{1}\right), \mathbf{a}\left(\theta_{2}\right), \ldots, \mathbf{a}\left(\theta_{K}\right)\right]$ is an $N \times K$ matrix whose columns are steering vectors of the sources. The $N \times 1$ steering vector $\mathbf{a}\left(\theta_{k}\right)$ models the spatial response of the array due to an incident plane wave from the $\theta$ direction and is given as [12]

$$
\mathbf{a}\left(\theta_{k}\right)=\left[\begin{array}{llll}
1 & e^{-j \mathcal{K} d \sin \theta_{k}} & \cdots & e^{-j \mathcal{K} d(N-1) \sin \theta_{k}}
\end{array}\right]^{T} .
$$

The array correlation matrix associated with vector $\mathbf{x}(t)$ contains information about how signals from each element are correlated with each other and is given by

$$
\mathbf{R}_{x x}=E\left[\mathbf{x}(t) \mathbf{x}^{H}(t)\right]
$$


where $E[\cdot]$ denotes expectation or statistical averaging operator and $(\cdot)^{H}$ denotes Hermitian transpose.

Let $s_{1}(t)$ be the desired signal source arriving from direction $\theta_{1}$ and consider the rest of the signals $s_{k}(t), k=2,3, \ldots, K$, as interferences arriving from their respective directions. The array output is given by

$$
y(t)=\mathbf{w}^{H} \mathbf{x}(t)
$$

where $\mathbf{w}$ is the weight vector that is applied to the antenna array to produce a beam pattern with its main lobe in the direction of the desired user. Assuming that maximum signal-to-nosie ratio beamforming is performed, $\mathrm{w}$ is given by

$$
\mathbf{w}=\eta_{1} \mathbf{v}_{\max }
$$

where $\mathbf{v}_{\max }$ is the eigenvector corresponding to the largest eigenvalue $\lambda_{\max }$ of $\mathbf{R}_{x x}$. It was shown in [5] that the eigenvector corresponding to the maximum eigenvalue of the array correlation matrix is approximately equal to the steering vector of the desired user when the desired signal is much stronger than the interferers at the receiver. Thus $\mathbf{w}$ is given by

$$
\mathbf{w}=\eta_{1} \mathbf{a}\left(\theta_{1}\right)
$$

where $\eta_{1}$ is a constant and is set to $1 / N$. Thus the array becomes the phased array as the magnitudes of the weight vector are constant and only the phases are varying. Substituting (7) in (5) and using (2) and simplifying, we get

$$
y(t)=s_{1}(t)+\frac{1}{N} \sum_{k=2}^{K} s_{k}(t) \mathbf{a}^{H}\left(\theta_{1}\right) \mathbf{a}\left(\theta_{k}\right)+\frac{1}{N} \mathbf{a}^{H}\left(\theta_{1}\right) \mathbf{n}(t) .
$$

The mean output power of the processor is

$$
\begin{aligned}
P(t)= & E\left[y(t) y^{*}(t)\right] \\
= & E\left[\left|s_{1}(t)\right|^{2}\right]+\sum_{k=2}^{K} \frac{1}{N^{2}}\left|\mathbf{a}^{H}\left(\theta_{1}\right) \mathbf{a}\left(\theta_{k}\right)\right|^{2} E\left[\left|s_{k}(t)\right|^{2}\right] \\
& +E\left[\frac{1}{N}\left|\mathbf{a}^{H}\left(\theta_{1}\right) \mathbf{n}(t)\right|^{2}\right] \\
= & E\left[\left|s_{1}(t)\right|^{2}\right]+\sum_{k=2}^{K} \alpha_{k}\left(\theta_{1}, \theta_{k}\right) E\left[\left|s_{k}(t)\right|^{2}\right]+\frac{\sigma^{2}}{N} .
\end{aligned}
$$

The first term on the right side of (9) is the desired signal power, whereas the second and third terms represent interference and noise power, respectively.

\section{SPATIAL INTERFERENCE SUPPRESSION COEFFICIENT}

The coefficient $\alpha_{k}\left(\theta_{1}, \theta_{k}\right)=\left(1 / N^{2}\right)\left|\mathbf{a}^{H}\left(\theta_{1}\right) \mathbf{a}\left(\theta_{k}\right)\right|^{2}$ in (9) is a measure of how much undesired power is picked up from an interferer. This is due to the fact that the array is unable to form a perfect pencil beam radiation pattern toward the desired signal at $\theta=\theta_{1}$ so the side lobes pick up interfering signals.

The normalized amount of interference power seen from an interferer $k$ at angle of arrival $\theta_{k}$ can be expressed in more general form as a scalar product of beamforming weight vector representing phased antenna elements and the array steering vector representing a plane wave as

$$
\alpha_{k}\left(\theta_{1}, \theta_{k}\right)=\left|\frac{\mathbf{w}^{H} \mathbf{a}\left(\theta_{k}\right)}{\left\|\mathbf{w}^{H}\right\|\left\|\mathbf{a}\left(\theta_{k}\right)\right\|}\right|^{2}
$$

where $\|(\cdot)\|$ denotes the Euclidean norm of a vector. Substituting the values from (3) and (7) and simplifying, we get

$$
\alpha_{k}\left(\theta_{1}, \theta_{k}\right)=\frac{1}{N^{2}}\left|\mathbf{a}^{H}\left(\theta_{1}\right) \mathbf{a}\left(\theta_{k}\right)\right|^{2}
$$

$$
=\frac{1}{N^{2}} \frac{\mid \sin \left(\left.\frac{\pi N}{2}\left(\sin \theta_{1}-\sin \theta_{k}\right)\right|^{2}\right.}{\left|\sin \left(\frac{\pi}{2}\left(\sin \theta_{1}-\sin \theta_{k}\right)\right)\right|^{2}} .
$$

Assuming the interferers are uniformly distributed in the range $[-\pi / 2, \pi / 2]$, the mean value of $\alpha_{k}\left(\theta_{1}, \theta_{k}\right)$ is given as

$$
G_{\mathrm{avg}}\left(\theta_{1}\right)=E\left[\alpha_{k}\left(\theta_{1}, \theta_{k}\right)\right]=\frac{1}{\pi} \int_{-\frac{\pi}{2}}^{\frac{\pi}{2}} \alpha_{k}\left(\theta_{1}, \theta_{k}\right) d \theta_{k}
$$

where $G_{\text {avg }}\left(\theta_{1}\right)$ is the spatial interference suppression coefficient [3].

Using (9), the instantaneous SIR at the array output $\left(\mathrm{SIR}_{\circ}\right)$ can be written as

$$
\operatorname{SIR}_{\mathrm{o}}=\frac{E\left[\left|s_{1}(t)\right|^{2}\right]}{\sum_{k=2}^{K} \alpha_{k}\left(\theta_{1}, \theta_{k}\right) E\left[\left|s_{k}(t)\right|^{2}\right]} .
$$

We see that the SIR at the array output is a function of $\theta_{1}$, the direction of the desired user. The mean SIR at the array output $\left(\operatorname{SIR}_{\circ}\right)$ can be written in terms of input SIR $\left(\mathrm{SIR}_{\text {in }}\right)$ as

$$
\operatorname{SIR}_{\mathrm{o}}=\frac{\operatorname{SIR}_{\text {in }}}{G_{\mathrm{avg}}\left(\theta_{1}\right)} .
$$

The average improvement in $\operatorname{SIR}(\Delta)$ at the array output is then given as

$$
\Delta=10 \log _{10}\left(\frac{1}{G_{\mathrm{avg}}\left(\theta_{1}\right)}\right)=-10 \log _{10}\left(G_{\mathrm{avg}}\left(\theta_{1}\right)\right) .
$$

\section{Circular ARray}

Next we consider a circular array of $N$ elements evenly spaced on a circle of radius $R=\lambda /[4 \sin (\pi / N)]$ in the $x y$-plane. This radius is chosen to maintain an interelement spacing of $d=\lambda / 2$, equivalent to that used for the linear array [13]. For convenience, we select the center of the circle as the phase reference. The array steering vector can then be written as

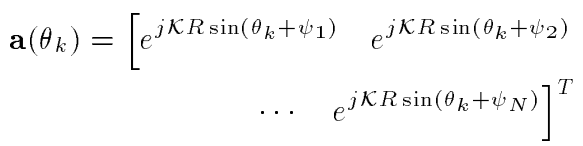

where $\psi_{n}=2 \pi(n-1) / N$ for $n=1,2, \ldots, N$ is the angular position of the $n$th element on the $x y$-plane. Assuming the interferers are uniformly distributed in the range $[-\pi, \pi], G_{\mathrm{avg}}\left(\theta_{1}\right)$ is given by

$$
G_{\mathrm{avg}}\left(\theta_{1}\right)=\frac{1}{2 \pi} \int_{-\pi}^{\pi} \alpha_{k}\left(\theta_{1}, \theta_{k}\right) d \theta_{k}
$$

where $\alpha_{k}\left(\theta_{1}, \theta_{k}\right)$ is given by (10) as before, with the steering vector given by (16).

\section{Mutual Coupling}

In order to include effect of mutual coupling for both linear and circular arrays, we insert a mutual coupling matrix in the model for the received signal, modifying (2) to

$$
\mathbf{x}(t)=\sum_{k=1}^{K} \mathbf{C} \mathbf{a}\left(\theta_{k}\right) s_{k}(t)+\mathbf{n}(t)=\sum_{k=1}^{K} \mathbf{a}_{M C}\left(\theta_{k}\right) s_{k}(t)+\mathbf{n}(t)
$$

where $\mathbf{a}_{M C}\left(\theta_{k}\right)=\mathbf{C} \mathbf{a}\left(\theta_{k}\right)$ is the modified array steering vector.

In addition, the beamforming weight vector is modified as

$$
\mathbf{w}_{M C}=\frac{1}{N} \mathbf{C} \mathbf{a}\left(\theta_{1}\right) \text {. }
$$


The coupling matrix $\mathbf{C}$ of the antenna array employed in the above two equations can be written using fundamental electromagnetics and circuit theory [14] as

$$
\mathbf{C}=\left(Z_{A}+Z_{T}\right)\left(\mathbf{Z}+Z_{T} \mathbf{I}_{N}\right)^{-1}
$$

where $Z_{A}$ is the element's impedance in isolation (for $\lambda / 2$ dipole, its value is $\left.Z_{A}=73+j 42.5[\Omega]\right), Z_{T}$ is the impedance of the receiver at each element and is chosen as the complex conjugate of $Z_{A}$ to obtain an impedance match for maximum power transfer, $\mathbf{I}_{N}$ is the identity matrix, and $\mathbf{Z}$ is the mutual impedance matrix given by

$$
\mathbf{Z}=\left(\begin{array}{cccc}
Z_{A}+Z_{T} & Z_{12} & \ldots & Z_{1 n} \\
Z_{21} & Z_{A}+Z_{T} & \ldots & Z_{2 n} \\
\vdots & \vdots & \vdots & \vdots \\
Z_{n 1} & Z_{n 2} & \ldots & Z_{A}+Z_{T}
\end{array}\right) .
$$

In general, numerical techniques such as method of moments can be used to obtain the mutual impedance matrix $\mathbf{Z}$. For dipoles, however, $\mathbf{Z}$ can be determined using classical induced electromotive force (EMF) method. For the side-by-side configuration and dipole lengths $l=\lambda / 2$, an element of the mutual impedance matrix $Z_{m n}$, where $1 \leq m, n \leq$ $N$, is given by [15]

$Z_{m n}$

$$
= \begin{cases}30\left[0.5772+\ln (2 \mathcal{K} l)-C_{i}(2 \mathcal{K} l)\right] & \\ \quad+j\left[30\left(S_{i}(2 \mathcal{K} l)\right)\right], & m=n(22 \mathrm{a}) \\ 30\left[2 C_{i}\left(u_{0}\right)-C_{i}\left(u_{1}\right)-C_{i}\left(u_{2}\right)\right] & \\ \quad-j\left[30\left(2 S_{i}\left(u_{0}\right)-S_{i}\left(u_{1}\right)-S_{i}\left(u_{2}\right)\right)\right], & m \neq n(22 \mathrm{~b})\end{cases}
$$

where $u_{0}=\mathcal{K} d_{h}, u_{1}=\mathcal{K}\left(\sqrt{d_{h}^{2}+l^{2}}+l\right), u_{2}=\mathcal{K}\left(\sqrt{d_{h}^{2}+l^{2}}-l\right)$, and $d_{h}$ is the horizontal distance between the two dipole antennas. $C_{i}(u)$ and $S_{i}(u)$ are the cosine and sine integrals, respectively, and are defined as $C_{i}(u)=\int_{\infty}^{u}(\cos (x) / x) d x$ and $S_{i}(u)=\int_{0}^{\infty}(\sin (x) / x) d x$. For comparison, we also use FEKO [16], which is a commercially available electromagnetic analysis package based on the method of moments, to determine the impedance matrix $\mathbf{Z}$ (in FEKO simulations, a frequency of $2 \mathrm{GHz}$ and a wire radius of $0.5 \mathrm{~mm}$ or $3.33 \times 10^{-3} \lambda$ are assumed).

Once the $\mathbf{C}$ matrix has been obtained, the capability of the array including mutual coupling effects be assessed by finding the mean output power of the processor, as before, and identifying the signal, noise, and interference power terms, respectively. It can be shown that with mutual coupling matrix taken into account, the normalized amount of interference power seen from an interferer $k$ at angle of arrival $\theta_{k}$ is

$$
\alpha_{k}\left(\theta_{1}, \theta_{k}\right)=\left|\frac{\mathbf{w}_{M C}^{H} \mathbf{a}_{M C}\left(\theta_{k}\right)}{\left\|\mathbf{w}_{M C}^{H}\right\|\left\|\mathbf{a}_{M C}\left(\theta_{k}\right)\right\|}\right|^{2}
$$

where $\mathbf{w}_{M C}$ is given by (19) and $\mathbf{a}_{M C}\left(\theta_{k}\right)$ by (18), respectively. The numerator in (23) after substituting the values becomes

$$
\begin{aligned}
\left|\mathbf{w}_{M C}^{H} \mathbf{a}_{M C}\left(\theta_{k}\right)\right|^{2} & =\left|\left[\frac{1}{N} \mathbf{C} \mathbf{a}\left(\theta_{1}\right)\right]^{H}\left[\mathbf{C} \mathbf{a}\left(\theta_{k}\right)\right]\right|^{2} \\
& =\frac{1}{N^{2}}\left|\mathbf{a}^{H}\left(\theta_{1}\right) \mathbf{C}^{H} \mathbf{C} \mathbf{a}\left(\theta_{k}\right)\right|^{2} .
\end{aligned}
$$

Equation (23) is then substituted in (12) and (17) to get the spatial interference suppression coefficient for linear and circular arrays, respectively.

\section{RESULTS}

When there is no mutual coupling, the matrix $\mathbf{Z}$ appearing in (21) is diagonal. In the presence of mutual coupling between antenna elements, there are nonzero elements off the diagonal. Fig. 1 shows the plot of the magnitude of the normalized impedance matrix elements
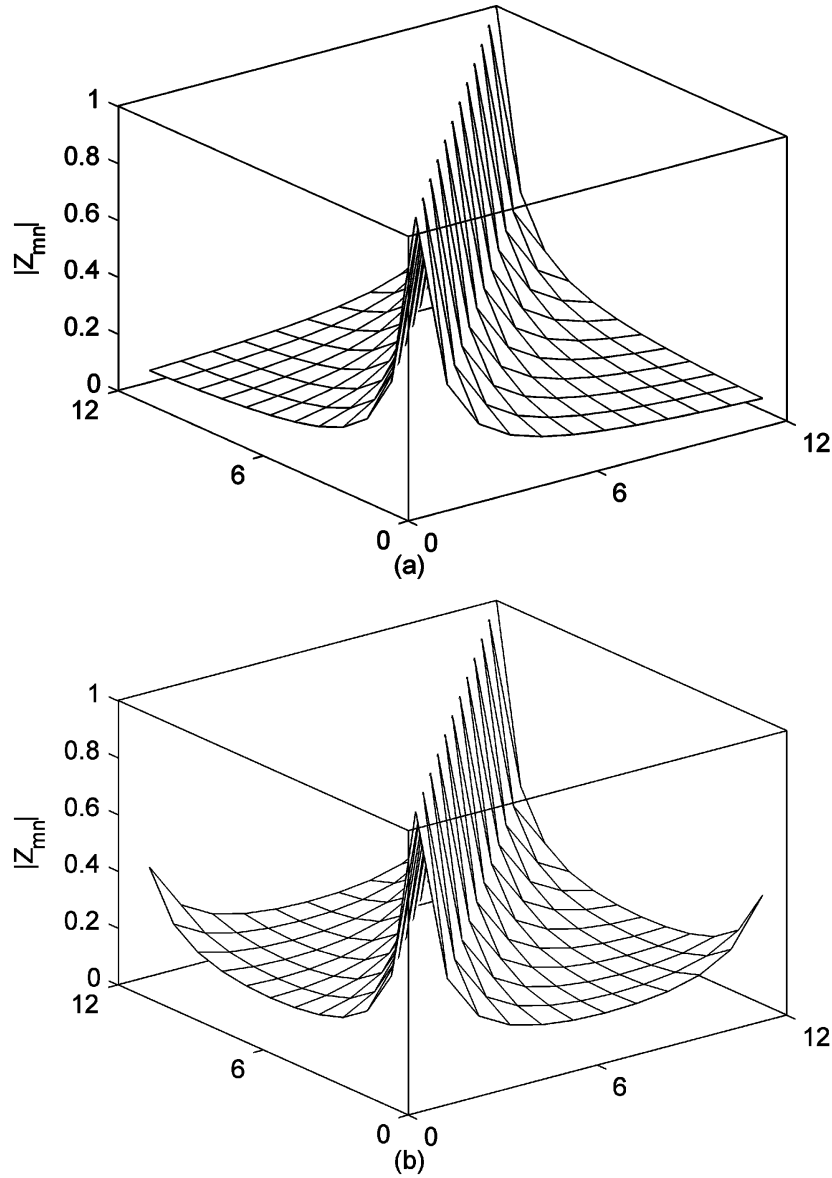

Fig. 1. Magnitude of the normalized impedance matrix elements for an array of $\boldsymbol{N}=\mathbf{1 2}, \boldsymbol{l}=\boldsymbol{\lambda} / \mathbf{2}$ dipoles with (a) linear and (b) circular geometries.

for an array of $N=12, l=\lambda / 2$ dipoles with terminating impedance $Z_{T}=Z_{A}$ for (a) linear and (b) circular geometries, respectively. The figure shows that for a linear array, the coupling between the neighboring elements is almost the same along the array. Also the magnitude of the coupling decreases quite rapidly as we move away from the diagonal. For a circular array, the magnitude also decreases initially but it increases again due to the circular symmetry of the geometry.

Fig. 2 shows a plot of the spatial interference suppression coefficient $G_{\text {avg }}\left(\theta_{1}\right)$ for a linear array for different number of antenna elements $N$, without and with mutual coupling. The curves are U-shaped, with a broad minimum, implying that interference reduction is maximum over a certain range of $\theta_{1}$, centered at $\theta_{1}=0^{\circ}$ (broadside). We can gain more insight into the interference suppression capability by plotting the average improvement in $\operatorname{SIR}(\Delta)$ versus the direction of arrival (DOA). This is shown in Fig. 3 for an $N=8$ element linear array under no mutual coupling assumption. We see that the exact amount of SIR at the array output is dependent on $\theta_{1}$. The maximum SIR improvement of about $10.85 \mathrm{~dB}$ is achieved when DOA is $\theta=0^{\circ}$. However, this decreases by more than half to about $5.1 \mathrm{~dB}$ for end-fire incidence. The presented result confirms our earlier expectation that for a linear array, its discrimination against interferers (in terms of SIR) is best in the array's broadside direction and deteriorates in its end-fire directions. To characterize this degradation in performance as we move from broadside to end-fire direction, we can define an interference reduction beamwidth $\left(\mathrm{BW}_{i r}\right)$ as the range of $\theta_{1}$ over which $G_{\mathrm{avg}}\left(\theta_{1}\right)$ is within $3 \mathrm{~dB}$ of its maximum value at $0^{\circ}$. Fig. 4 shows the variation of $\mathrm{BW}_{\text {ir }}$ with $N$. For $N=4$, the beamwidth is approximately $\pm 51^{\circ}$ but increases to about $\pm 59^{\circ}$ for $N=12$. The range is nearly the same when 


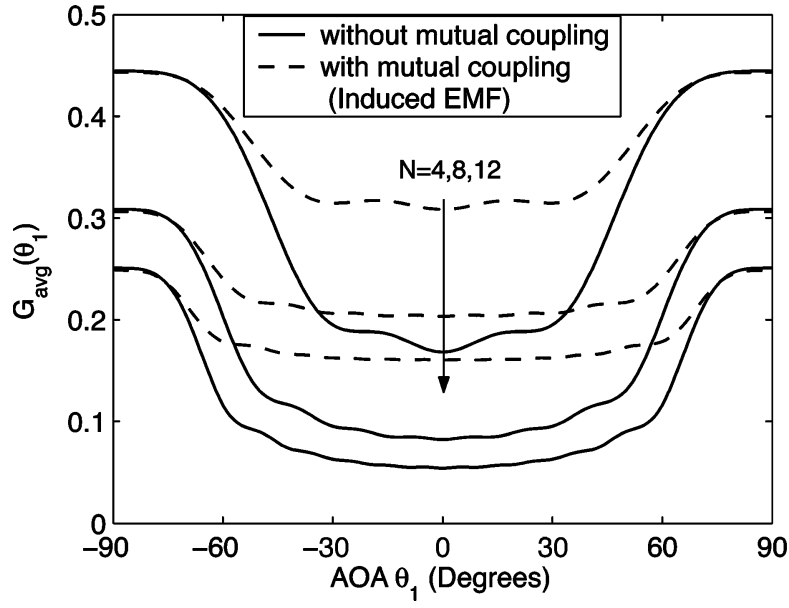

Fig. 2. Variation of the spatial interference coefficient $\boldsymbol{G}_{\mathrm{avg}}\left(\boldsymbol{\theta}_{1}\right)$ with $\boldsymbol{\theta}_{1}$ for linear array $(N=4,8,12)$.

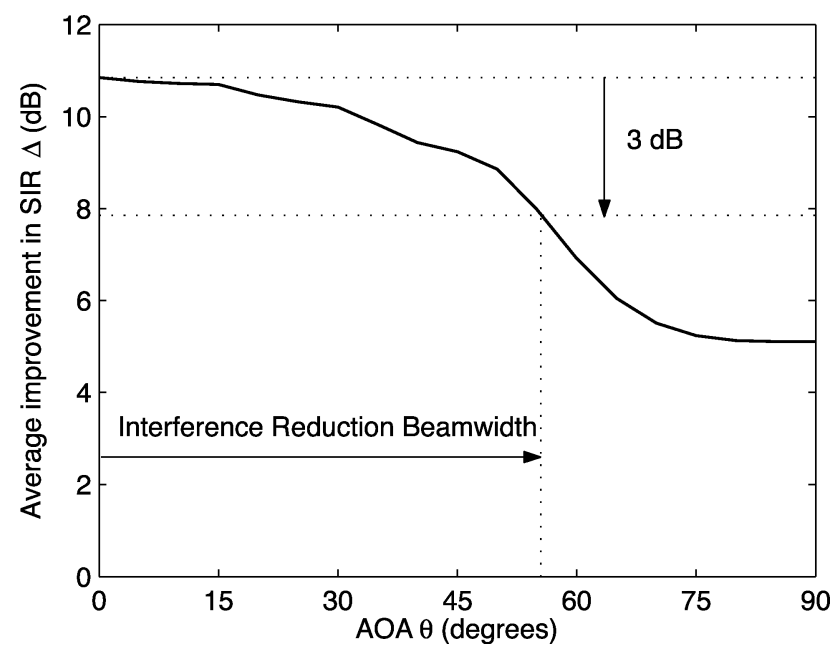

Fig. 3. Plot of average improvement in SIR versus AOA for $\mathbf{N}=\mathbf{8}$ element linear array, for the case of no mutual coupling.

$N$ increases further, with a small oscillation about this value, implying the onset of diminishing returns.

Fig. 2 also shows that for a linear array, mutual coupling degrades the SIR improvement capability of the array. For larger scan angles, the performance is the same as for the case of no mutual coupling, but for broadside incidence there is degradation due to mutual coupling.

The plot of $G_{\mathrm{avg}}\left(\theta_{1}\right)$ for a circular array is shown in Fig. 5. For a smaller number of antenna elements, the SIR improvement shows an oscillatory variation. However, as $N$ increases the curves flatten, indicating uniform SIR improvement over all angles. Contrary to the case of the linear array, a slight improvement in performance can be observed when mutual coupling is included, for all scan angles. The figure also shows that the curves obtained using $\mathbf{Z}$ determined from the induced EMF method show a good agreement with the curves obtained using $\mathbf{Z}$ calculated from FEKO. The slight difference in the curves can be attributed to limitations of the induced EMF method, e.g., it cannot take into account the gaps at the feed and the radius of the wires.

Finally, Table I shows a comparison of mean of $G_{\text {avg }}\left(\theta_{1}\right)$ over $\theta_{1}$ for different $N$ for linear and circular arrays, with and without mutual coupling. The mean is taken over $180^{\circ}(-\pi / 2 \leq \theta \leq \pi / 2)$ for linear array and $360^{\circ}(-\pi \leq \theta \leq \pi)$ for circular array. The values confirm

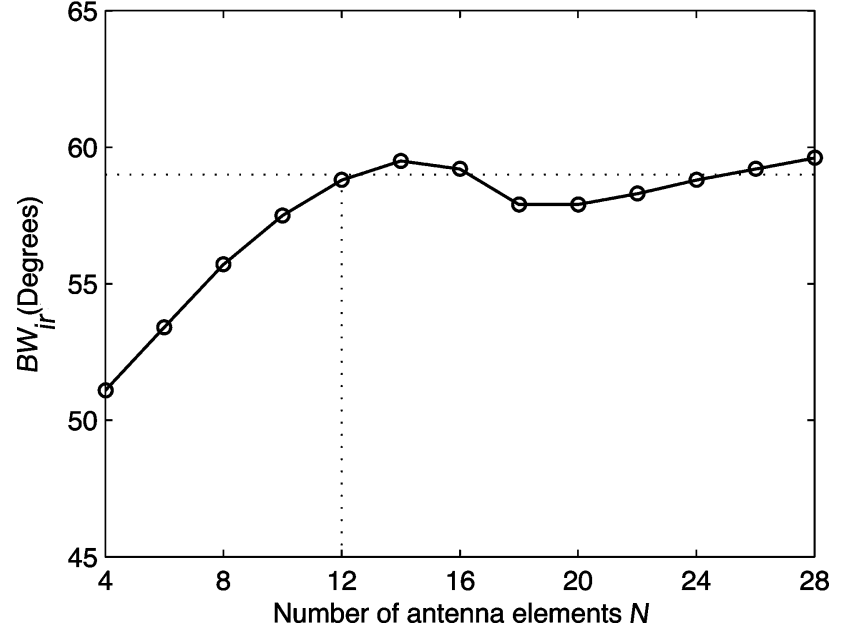

Fig. 4. Plot of interference reduction beamwidth with number of antenna elements $\boldsymbol{N}$ for a linear array under no mutual coupling assumption.

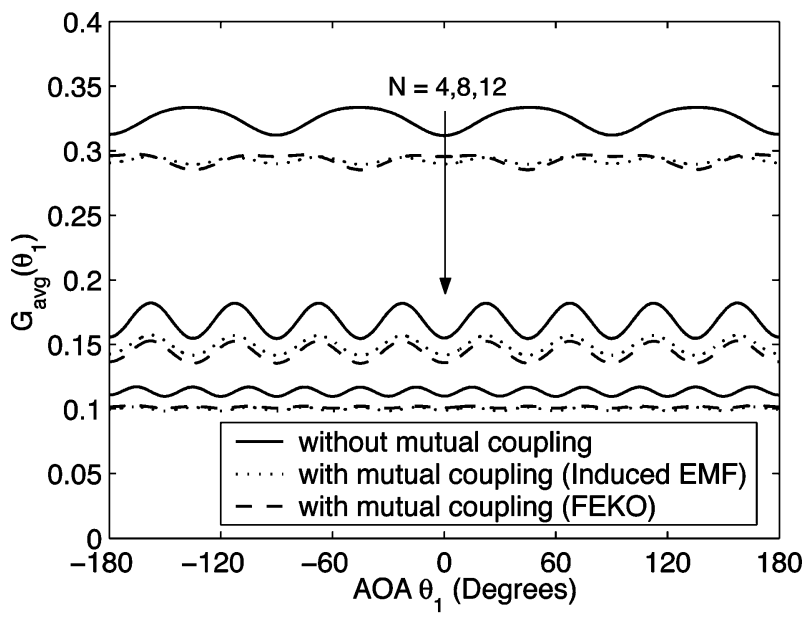

Fig. 5. Variation of the spatial interference suppression coefficient $\boldsymbol{G}_{\mathrm{avg}}\left(\boldsymbol{\theta}_{1}\right)$ with $\boldsymbol{\theta}_{1}$ for circular array $(N=4,8,12)$.

TABLE I

MEAN OF $\boldsymbol{G}_{\mathrm{avg}}\left(\boldsymbol{\theta}_{1}\right)$ OVER $\boldsymbol{\theta}_{1}$ FOR LINEAR AND CIRCULAR ARRAYS, With AND Without MUtUAL COUPLING

\begin{tabular}{c|c|c|c|c}
\hline \multirow{2}{*}{$N$} & \multicolumn{4}{|c}{ Average of $G_{a v g}\left(\theta_{1}\right)$ over $\theta_{1}$} \\
\cline { 2 - 5 } & \multicolumn{2}{|c}{ Linear } & \multicolumn{2}{c}{ Circular } \\
\cline { 2 - 5 } & without coupling & with coupling & without coupling & with coupling \\
\hline 4 & 0.3021 & 0.3667 & 0.3241 & 0.2926 \\
8 & 0.1668 & 0.2369 & 0.1683 & 0.1438 \\
12 & 0.1176 & 0.1852 & 0.1134 & 0.0996 \\
16 & 0.0917 & 0.1558 & 0.0915 & 0.0811 \\
20 & 0.0756 & 0.1363 & 0.0774 & 0.0722 \\
\hline
\end{tabular}

that for large $N$, the interference rejection capability reaches an asymptotic level. For a linear array, the values when mutual coupling is taken into account are higher, again illustrating the performance degradation. However, for a circular array, the values when mutual coupling is taken into account are slightly improved. 


\section{CONCLUSION}

This paper has provided and compared results for the average SIR improvement for linear and circular arrays of half-wavelength spaced dipoles, which can be part of a base station of a CDMA cellular communication system. Two cases, one where mutual coupling is neglected and a second where mutual coupling is included, have been considered. It has been shown that there is an improvement in SIR as $N$ increases. For a linear array, the range of angles over which the most significant improvement occurs is limited to approximately $\pm 59^{\circ}$. This occurs when about 12 antenna elements are used. Increasing $N$ further results in diminishing returns. In comparison, for a full angular range surrounding a base station, a circular array provides a more uniform improvement in terms of SIR than a linear array. Also the obtained results have shown that mutual coupling degrades the SIR improvement capability of the linear array, particularly in the broadside direction. In contrast, mutual coupling has little effect on the SIR improvement capability of the circular array for the assumed element spacing of half-wavelength.

\section{ACKNOWLEDGMENT}

The authors would like to thank the anonymous reviewers for their constructive suggestions that helped improve the quality of this paper.

\section{REFERENCES}

[1] G. V. Tsoulos, Ed., Adaptive Antennas for Wireless Communications. New York: IEEE Press, 2001.

[2] T. S. Rappaport, Ed., Smart Antennas: Adaptive Arrays, Algorithms \& Wireless Position Location. New York: IEEE Press, 1998.
[3] Y. S. Song and H. M. Kwon, "Analysis of a simple smart antenna for CDMA wireless communications," in Proc. IEEE Vehicular Technology Conf., San Antonio, TX, May 16-20, 1999, pp. 254-258.

[4] R. S. Adve and T. K. Sarkar, "Compensation for the effects of mutual coupling on direct data domain adaptive algorithms," IEEE Trans. Antennas Propagat., vol. 48, pp. 86-94, Jan. 2000.

[5] S. Choi and D. Yun, "Design of adaptive antenna array for tracking the source of maximum power and its application to CDMA mobile communications," IEEE Trans. Antennas Propagat., vol. 45, pp. 1393-1404, Sept. 1997

[6] G. W. K. Colman and S. D. Blostein, "Improved power and capacity predictions of a CDMA system with base-station antenna arrays and digital beamforming," in Proc. 19th Biennial Symp. Communications, Kingston, June 1998, pp. 280-284.

[7] S. Durrani and M. E. Bialkowski, "Investigation into the performance of a adaptive array in cellular environment," in Proc. IEEE AP-S Int. Symp., vol. 2, San Antonio, TX, June 16-21, 2002, pp. 648-651.

[8] Y. S. Song, H. M. Kwon, and B. J. Min, "Computationally efficient smart antennas for CDMA wireless communications," IEEE Trans. Veh Technol., vol. 50, pp. 1613-1628, Nov. 2001.

[9] A. M. Wyglinski and S. D. Blostein, "Mutual coupling and scattering effects on cellular CDMA systems using smart antennas," in Proc. IEEE VTC 2000 Fall, vol. 4, Boston, MA, Sept. 2000, pp. 1656-1662.

[10] A. M. Wyglinski and S. D. Blostein, "On uplink CDMA cell capacity: mutual coupling and scattering effects on beamforming," IEEE Trans. Veh. Technol., vol. 52, pp. 289-304, Mar. 2003.

[11] T. Svantesson and A. Ranheim, "Mutual coupling effects on the capacity of multielement antenna systems," in Proc. IEEE ICASSP 01, vol. 4, Salt Lake City, UT, May 2001, pp. 2485-2488.

[12] Handbook of Antennas in Wireless Communications, L. C. Godara, Ed., CRC Press, Boca Raton, FL, 2002.

[13] R. A. Monzingo and T. W. Miller, Introduction to Adaptive Arrays. New York: Wiley, 1980.

[14] T. Svantesson, "Modeling and estimation of mutual coupling in a uniform linear array of dipoles," in Proc. ICASSP'99, Phoenix, AZ, Mar 1999, pp. 2961-2964.

[15] C. A. Balanis, Antenna Theory Analysis and Design. New York: Wiley, 1997.

[16] FEKO User's Manual, EM Software \& Systems Inc., July 2001. 